\title{
DYNAMICS OF MATHEMATICAL MODELS FOR BIOREACTORS
}

\author{
ZHENZHEN CHEN
}

(Received 10 July 2017; first published online 28 September 2017)

\section{Mathematics subject classification: primary 34D20; secondary 34C60. \\ Keywords and phrases: bioreactors, mathematical modelling, dynamics, stability.}

Bioreactors are vessels in which a biological reaction takes place. They are ideal apparatuses to model many natural phenomena in ecosystems. Bioreactors are used extensively in the biological treatment of wastes and the manufacture of useful bioproducts. If designed and operated correctly, a bioreactor can be a relatively cost-effective method for hazardous substance removal and bioproduct manufacture. Mathematical models are valuable tools to study bioreactors [3], since they are mathematically tractable and the relevant experiments can be carried out. Therefore, bioreactor modelling has great potential for use in many fields, such as to assist in the design of equipment, to predict long-term behaviour, to interpret data and to control and optimise the bioreactor.

The objectives of this thesis are to develop mathematical models for bioreactors by capturing essential information on bioreactors and then to analyse the developed models to predict the performance of bioreactors, which may provide a useful aid for improving the performance of bioreactors.

The goal of biological wastewater treatment is to use microorganisms to remove organic materials and pollutants in wastewater, since microorganisms consume organic materials/pollutants to grow. Biological wastewater treatment in flow bioreactors has been intensively studied. However, the majority of previous work has invariably assumed that all the parameters in wastewater treatment models such as the recycle rate and the death rate of microorganisms are constants, whereas the fact is that there might be different ways in which uncertainties can influence wastewater treatment processes in practice. The parameters in the model may fluctuate due to environmental variations such as air pressure and temperature fluctuations, and this fact can be described by stochastic models. Motivated by this, in the first part of the thesis, stochastic

Thesis submitted to Swinburne University of Technology in October 2016; degree approved on 8 March 2017; principal supervisor Tonghua Zhang, cosupervisors Moses Tade and Geoffrey Brooks.

(C) 2017 Australian Mathematical Publishing Association Inc. 0004-9727/2017 \$16.00 
models for both single bioreactors and cascaded bioreactors used in wastewater treatment processes are developed. The long-term behaviours of stochastic models are studied and compared with those of their deterministic counterparts. We also use numerical simulations to investigate the effect of noise on the performance of the bioreactors. Furthermore, to improve the performance of the bioreactors, a control strategy of introducing a state-dependent feedback to the model for biological wastewater treatment is proposed, which involves the application of an amount of microorganisms to the wastewater once the pollutant concentration is higher than a certain threshold. This results in a state-dependent impulsive model. The long-term dynamics of the impulsive model are then investigated mathematically [2]. Our study shows that our new model outperforms the traditional models. Most importantly, this control strategy is applicable in practice and cost effective.

In the second part of the thesis, a modified chemostat called a morbidostat is studied. A morbidostat is a bacteria culture device that maintains a nearly constant microbial population for the selection of drug-resistant mutants via a feedback algorithm. This device has been used intensively in cultivating microbes [4]. However, to date no mathematical analysis has been demonstrated for the morbidostat to the best of our knowledge. In this part, we propose mathematical models of morbidostats when a microbial species undergoes sequential evolution [1]. The models are studied in detail to elucidate the operation of a morbidostat. The cultivation of microbes is assumed to be under periodic dilution, and a simple threshold algorithm is used as feedback. The theoretical framework elucidates the generic features of the operation of a morbidostat under drug-inhibitor-induced feedback, and will provide a useful aid in the design of experiments.

The full thesis is available at https://researchbank.swinburne.edu.au/file/073671bbcf7c-4b6f-bdfc-a805789a20b5/1/Zhenzhen.

\section{References}

[1] Z. Chen, S. B. Hsu and Y. T. Yang, 'The morbidostat: a bio-reactor that promotes selection for drug resistance in bacteria', SIAM J. Appl. Math. 77(2) (2017), 470-499.

[2] Z. Chen, T. Zhang, M. O. Tade and G. Brooks, 'Existence of periodic solutions of a continuous flow bioreactor model with impulsive control in microorganisms', J. Appl. Math. Comput. 53(1) (2017), $471-486$.

[3] H. L. Smith and P. E. Waltman, The Theory of the Chemostat (Cambridge University Press, Cambridge, 1995).

[4] E. Toprak, A. Veres, J. B. Mitchel, D. L. Hartl and R. Kishony, 'Evolutionary paths to antibiotic resistance under dynamically sustained drug selection', Nature Genetics 44 (2012), 101-106.

\footnotetext{
ZHENZHEN CHEN, Department of Mathematics, Swinburne University of Technology, Victoria 3122, Australia e-mail: zhenzhenchen1221@yahoo.com
} 